\title{
Methods of ESBLs Detection in Clinical Microbiology Lab
}

\section{Abdelrahman Abdelmoktader ${ }^{1 *}$ and Amira Talal El Far ${ }^{2}$ \\ ${ }^{1}$ Lecture of medical microbiology and immunology, Fayoum University, Egypt \\ ${ }^{2}$ Researcher assistant of clinical pathology, Theodor Bilharz Research Institute, Egypt}

*Corresponding author: Abdelrahman Abdelmoktader, Lecture of medical microbiology and immunology, Faculty of medicine, Fayoum University, Egypt, Email: aam16@fayoum.edu.eg

\section{Review Article \\ Volume 3 Issue 4}

Received Date: October 02, 2019

Published Date: October 18, 2019

DOI: $10.23880 / \mathrm{vij}-16000222$

\section{Abstract}

Microbial resistance through extended-spectrum $\beta$-lactamase (ESBL) has emerged globally, and ESBL -producing Enterobacteriacae are recognized worldwide as nosocomial pathogens of major importance.

Several phenotypic tests have been recommended for screening and confirmation of ESBL-producing organisms, but these are usually performed on isolated organisms following culture and antibiotic susceptibility testing.

Keywords: Extended Spectrum Beta Lactamases; Phenotypic Methods; Broth Microdilution; Three Dimentional Test; Disc Diffusion Test; Double Disc Synergy Test; Combination Disc Test; E Test

\section{Introduction}

$\beta$-Lactamases are enzymes that inactivate $\beta$-lactam antibiotics by opening the $\beta$-lactam ring of penicillins and cephalosporins and abolish their antimicrobial activity. Beta-lactamases have been described for many species of gram-positive and gram-negative bacteria. Some $\beta$ lactamases are plasmid-mediated (e.g, penicillinase of Staphylococcus aureus), while others are chromosomally mediated (e.g, many species of gram-negative bacteria) [1].

The failure to detect ESBL-mediated resistance has led to treatment failure and contributed to uncontrolled spread of ESBL-producing organisms. On the other hand, laboratory-based detection of patients infected or colonized by ESBL-producing organisms by surveillance cultures has proven useful to control and terminate nosocomial outbreaks [2].

Many clinical microbiology laboratories have problems with the detection of ESBL-mediated resistance, and the recent emergence and spread of novel types of community-acquired ESBLs, have created additional challenges that further complicate the detection of this resistance mechanism. Several phenotypic tests have been recommended for screening and confirmation of ESBLs, but these are usually performed on isolated organisms following culture and antibiotic susceptibility testing [3].

ESBL detection originated because some ESBLproducing organisms appeared susceptible to cephalosporins using conventional breakpoints. How frequently are ESBL-producing organisms susceptible to cephalosporins? The answer to this question depends on which breakpoints are used. National differences are quite considerable. For example, susceptibility to cefotaxime may be reported for an organism with MICs ranging from $\leq 1 \mu \mathrm{g} / \mathrm{ml}$ to $\leq 8 \mu \mathrm{g} / \mathrm{ml}$, depending on the country. Variation in reporting of resistance is from $\geq 2 \mu \mathrm{g} / \mathrm{ml}$ to $\geq 64 \mu \mathrm{g} / \mathrm{ml}$. The most liberal interpretation of cephalosporin susceptibility has been that of the Clinical and Laboratory Standards Institute (CLSI, formerly 


\section{Virology \& Immunology Journal}

National Committee for Clinical Laboratory Standards) (cephalosporin susceptibility indicated by MICs $\leq 8 \mu \mathrm{g} / \mathrm{ml}$ ) (Table 2). The CLSI breakpoints for susceptibility of members of the Enterobacteriaceae to extended-spectrum cephalosporins and aztreonam were developed in the early 1980s. At that time the clinical success rate of cephalosporin treatment for organisms with cephalosporin MICs of $\leq 8 \mu \mathrm{g} / \mathrm{ml}$ (that is, organisms with MICs in the susceptible range) was $>95 \%$. Unfortunately these breakpoints were developed at a time that was essentially prior to the advent of ESBLs [4].

\begin{tabular}{|c|c|c|c|c|}
\hline \multirow{2}{*}{ Country } & \multicolumn{4}{|c|}{ 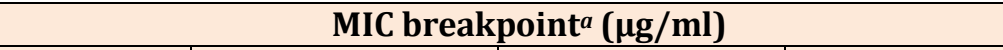 } \\
\hline & $S(\leq)$ & $\mathbf{R}(\geq)$ & $S(\leq)$ & $\mathbf{R}(\geq)$ \\
\hline United States of America (CLSI) & 8 & 64 & 8 & 32 \\
\hline United Kingdom & 1 & 2 & 2 & 4 \\
\hline France & 4 & 32 & 4 & 32 \\
\hline The Netherlands & 4 & 16 & 4 & 16 \\
\hline Germany & 2 & 8 & 4 & 32 \\
\hline Spain & 1 & 8 & 1 & 8 \\
\hline Norway & 2 & 16 & 2 & 16 \\
\hline Sweden & 4 & 32 & 4 & 16 \\
\hline
\end{tabular}

${ }^{a} \mathrm{~S}$, susceptible; R, resistant.

Table 1: Comparison of national MIC breakpoints for Enterobacteriaceae.

In a review of studies which have evaluated collections of ESBL-producing organisms using standard CLSI disc diffusion or MIC breakpoints, 13 to $49 \%$ of isolates were cefotaxime susceptible, 36 to $79 \%$ were ceftriaxone susceptible, 11 to $52 \%$ were ceftazidime susceptible, and 10 to $67 \%$ were aztreonam susceptible. Approximately $40 \%$ of tested organisms were susceptible to at least one oxyimino ß-lactam (e.g, cefotaxime, cefriaxone, ceftazidime) and $20 \%$ to all oxyimino ß-lactams. The reason for this apparent susceptibility to some cephalosporins is the result of various degrees of hydrolysis of cephalosporins by different ß-lactamases and enhanced penetration through the bacterial outer membrane of some cephalosporins compared to others. Regardless, extended-spectrum cephalosporin MICs of 2 to $8 \mu \mathrm{g} / \mathrm{ml}$ are 4 to 8 dilutions higher than those seen in the same strain producing only the parent TEM-1, TEM-2, or SHV-1 ß-lactamase (0.03 to $0.25 \mu \mathrm{g} / \mathrm{ml}$ ) [5].

It has been well recognized for some time that poor outcome occurs when patients with serious infections due to ESBL-producing organisms are treated with cephalosporins to which the organism is frankly resistant. The failure rate in such patients has ranged from 42 to $100 \%$. Similar failure rates exist when cephalosporins are used to treat patients with serious infections due to ESBL producers which have cephalosporin MICs in the intermediate range and even with some MICs in the susceptible range. The failure rate when cephalosporins were used for serious infections (bacteremia, hospitalacquired pneumonia and peritonitis) with ESBL- producing organisms with MICs for the treating cephalosporin of 4 to $8 \mu \mathrm{g} / \mathrm{ml}$ exceeds $90 \%$. The failure rate when MICs for the treating cephalosporin were $\leq 2$ $\mu \mathrm{g} / \mathrm{ml}$ is substantially lowers [6].

It is recommended by the CLSI that clinical microbiology laboratories perform specialized tests for detection of ESBLs. There are two reasons for this. First, there is value of the knowledge of presence of ESBLs in terms of aiding infection control. Second, in the presence of high-inoculum infections (e.g intra-abdominal abscess and some cases of pneumonia) or infections at sites in which drug penetration may be poor (e.g. meningitis, endocarditis, or osteomyelitis), physicians should avoid cephalosporins if an ESBL-producing organism is present [4].

\section{Screening Methods for ESBL Production}

\section{Disc Diffusion Method}

The CLSI has proposed disc diffusion methods for screening for ESBL production by klebsiellae, Escherichia coli, and Proteus mirabilis. Laboratories using disc diffusion methods for antibiotic susceptibility testing can screen for ESBL production by noting specific zone diameters which indicate a high level of suspicion for ESBL production. Cefpodoxime, ceftazidime, aztreonam, cefotaxime, or ceftriaxone may be used. However, the use of more than one of these agents for screening improves the sensitivity of detection. If any of the zone diameters indicate suspicion for ESBL production, phenotypic 
confirmatory tests should be used to ascertain the diagnosis [4].

Mention should be made of the use of cefpodoxime as a screening antibiotic since this antibiotic is not widely used in inpatient facilities [7]. Noted that cefpodoxime susceptibility by disc diffusion reliably discriminated between ESBL-producing and non-ESBL-producing Klebsiella pneumoniae and Escherichia coli. The CLSI initially recommended a zone diameter of $\leq 22 \mathrm{~mm}$ for a 10- $\mu$ g cefpodoxime disc as a suitable screening test for ESBL production. Unfortunately, the cefpodoxime screening test using a zone diameter of $\leq 22 \mathrm{~mm}$ lacks specificity when used to screen Escherichia coli isolates for ESBL production. Therefore, the CLSI now recommends a change in the cefpodoxime screening zone diameter to $\leq 17 \mathrm{~mm}$; that is, isolates with a cefpodoxime zone diameter of $\leq 17 \mathrm{~mm}$ should undergo phenotypic confirmatory tests for ESBL production [4].

\section{Dilution Antimicrobial Susceptibility Test}

The CLSI has proposed dilution methods for screening for ESBL production by klebsiellae and Escherichia coli. Ceftazidime, aztreonam, cefotaxime, or ceftriaxone can be used at a screening concentration of $1 \mu \mathrm{g} / \mathrm{ml}$. Growth at this screening antibiotic concentration (that is, MIC of the cephalosporin of $\geq 2 \mu \mathrm{g} / \mathrm{ml}$ ) is suspicious of ESBL production and is an indication for the organism to be tested by a phenotypic confirmatory test [4].

The originally proposed screening criterion for cefpodoxime was that isolates which were potential ESBL producers had cefpodoxime MIC of $\geq 2 \mu \mathrm{g} / \mathrm{ml}$. However, in a study of the mechanisms of decreased susceptibility of Escherichia coli to cefpodoxime, it was found that none of 59 strains with cefpodoxime MICs of 2 or $4 \mu \mathrm{g} / \mathrm{ml}$ produced ESBLs. The most common mechanism of reduced susceptibility to cefpodoxime was production of the TEM-1 ß-lactamase associated with the loss or alteration of a major porin protein. Other strains lacked production of TEM-1 but had porin changes, sometimes coupled with modest elevation in production of the AmpC chromosomal ß-lactamase. Finally, some of the isolates produced the OXA-30 ß-lactamase [8].

\section{Confirmatory Tests for ESBL Production}

\section{Phenotypic Tests}

Cephalosporin/Clavulanate Combination Discs

The CLSI advocates use of cefotaxime $(30 \mu \mathrm{g})$ or ceftazidime discs (30 $\mu \mathrm{g})$ with and without clavulanate $(10 \mu \mathrm{g})$ for phenotypic confirmation of the presence of

\section{Virology \& Immunology Journal}

ESBLs in klebsiellae and Escherichia coli. Prior to the combination discs becoming available, it was recommended that clavulanic acid solution be applied to the cephalosporin discs within one hour before they are applied to the agar plates. The CLSI recommends that the disc tests be performed with confluent growth on Mueller-Hintonagar. A difference of $\geq 5 \mathrm{~mm}$ between the zone diameters of either of the cephalosporin discs and their respective cephalosporin/clavulanate disc is taken to be phenotypic confirmation of ESBL production.

Another method known as the disc replacement method was described. Three amoxicillin/clavulanate discs are applied to a Mueller-Hinton plate inoculated with the test organism. After 1 hour at room temperature, these antibiotic discs are removed and replaced on the same spot by discs containing cefotaxime, ceftazidime and aztreonam. Control discs of these three antibiotics are simultaneously placed at least $30 \mathrm{~mm}$ from these locations. A positive test is indicated by a zone increase of $>5 \mathrm{~mm}$ for the discs which have replaced the amoxicillin/clavulanate discs compared to the control discs Unfortunately this method is not suitable for busy clinical microbiology laboratories given the need for a second step one hour after the initial plate inoculation [9].

A modification of this test, in which discs inoculated with clavulanic acid were used instead of the amoxicillinclavulanic acid discs, has been described. The results of this method were comparable to the double-disc synergy test [10].

\section{Broth Microdilution}

Phenotypic confirmatory testing can also be performed by broth microdilution assays using ceftazidime $(0.25$ to $128 \mu \mathrm{g} / \mathrm{ml})$, ceftazidime plus clavulanic acid $(0.25 / 4$ to $128 / 4 \mu \mathrm{g} / \mathrm{ml})$, cefotaxime $(0.25$ to $64 \mu \mathrm{g} / \mathrm{ml})$, and cefotaxime plus clavulanic acid $(0.25 / 4$ to $64 / 4 \mu \mathrm{g} / \mathrm{ml}$ ). It should be emphasized that both ceftazidime and cefotaxime should be used. Broth microdilution is performed using standard methods. Phenotypic confirmation is considered as a $\geq 3$-twofoldserial-dilution decrease in MIC of either cephalosporin in the presence of clavulanic acid compared to its MIC when tested alone [11].

\section{Double-Disc Diffusion Test}

Described a disc diffusion test in which synergy between cefotaxime and clavulanate was detected by placing a disc of amoxicillin/clavulanate $(20 \mu \mathrm{g} / 10 \mu \mathrm{g}$, respectively) and a disc of cefotaxime (30 $\mu \mathrm{g}), 30 \mathrm{~mm}$ 
apart (center to center) on an inoculated agar plate [12]. A clear extension of the edge of the cefotaxime inhibition zone toward the disc containing clavulanate keyhole like) was interpreted as synergy, indicating the presence of an ESBL; 30- $\mu \mathrm{g}$ antibiotic discs of ceftazidime, aztreonam, and ceftriaxone were also placed on the plate, $30 \mathrm{~mm}$ (center to center) from the amoxicillin/clavulanate disc, since sometimes this keyhole effect was not observed with cefotaxime but is with other ß-lactam antibiotics containing the oxyanion group.

Evaluation of the double-disc diffusion test against strains which have been genotypically confirmed to be ESBL producers or non-producers have revealed sensitivities of the method ranging from $79 \%$ to $97 \%$ and specificities ranging from $94 \%$ to $100 \%$. False-negative results have been observed with isolates harboring SHV-2, SHV-3, and TEM-12. In isolates which are suspicious for harbouring ESBLs but are negative using the standard distance of $30 \mathrm{~mm}$ between discs, the test should be repeated using closer (for example, $20 \mathrm{~mm}$ ) spacing [13].

A major advantage of the double-disc diffusion test is that the test is technically simple. However, the interpretation of the test is quite subjective. Sensitivity may be reduced when ESBL activity is very low, leading to wide zones of inhibition around the cephalosporin and aztreonam discs. This has been noted for Proteus mirabilis

\section{Agar Supplemented with Clavulanate}

Described a method by which Mueller-Hinton agarwas supplemented with $4 \mu \mathrm{g} / \mathrm{ml}$ of clavulanate. Antibiotic discs containing ceftazidime $(30 \mu \mathrm{g})$, cefotaxime $(30 \mu \mathrm{g})$, ceftriaxone $(30 \mu \mathrm{g})$, and aztreonam $(30 \mu \mathrm{g})$ were placed on the clavulanate containing agar and on regular clavulanate free Mueller-Hinton agar plates [14]. A difference in $ß$-lactam zone width of $\geq 10 \mathrm{~mm}$ on the two media was considered positive for ESBL production. A major drawback of the method is the need to freshly prepared clavulanate containing plates. The potency of clavulanic acid begins to decrease after $72 \mathrm{~h} \mathrm{[15].}$

\section{Three-Dimensional Test}

The three-dimensional test gives phenotypic evidence of ESBL-induced inactivation of extended-spectrum cephalosporins or aztreonam without relying on demonstration of inactivation of the ß-lactamases by a $ß$ lactamase inhibitor. In this test, the surface of the susceptibility plate is inoculated by standard methods for disc diffusion testing using a susceptible organism to the

\section{Virology \& Immunology Journal}

B-lactam antibiotic disc used in the test. A slit is cut in the agar $5 \mathrm{~mm}$ from the edge of the $ß$-lactam antibiotic disc in an outward radial direction or a spot is formed in the agar 7-8mm away from the $ß$-lactam antibiotic disc. The slit or the spot is filled with $25 \mu \mathrm{L}$ of a milky suspension (McFarland number 5 turbidity standards) of the tested strain in broth. After overnight incubation at $37^{\circ} \mathrm{C}$, the three dimensional test is interpreted positive when the inhibition zone around the ß-lactam antibiotic disc is distorted by the growth of the tested organism resulting in decreasing the diameter of the inhibition zone (Figure 1) [16].

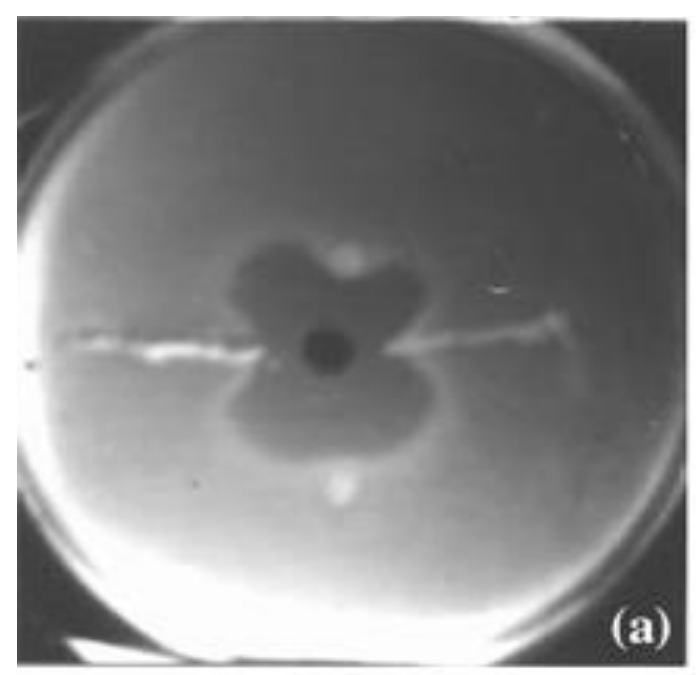

Figure 1: The Three-dimensional test.

\section{Commercial Methods for ESBL Detection}

\section{E-Test for ESBLs}

AB Biodisk (Solna, Sweden) produces plastic drugimpregnated strips, one end of which contains a gradient of ceftazidime (MIC test range 0.5 to $32 \mu \mathrm{g} / \mathrm{ml}$ ) and the other with a gradient of ceftazidime plus a constant concentration of clavulanate $(4 \mu \mathrm{g} / \mathrm{ml})$. Similar strips containing cefotaxime and cefotaxime/clavulanate are now available. The reported sensitivity of the method as a phenotypic confirmatory test for ESBLs is $87 \%$ to $100 \%$ and the specificity is $95 \%$ to $100 \%$. The sensitivity and specificity of the method depend on the ratio of MICs of the cephalosporin versus cephalosporin/clavulanate combination used. The manufacturer recommends a $\geq 8$ fold reduction in cephalosporin MICs in the presence of clavulanate. Occasionally the MIC of the cephalosporin alone is difficult to read because the inhibition zone is 


\section{Virology \& Immunology Journal}

distorted by the clavulanic acid diffusing from the opposite ends of the strip. In these cases, some prefer to measure the MIC of the cephalosporin alone using a separate conventional strip containing only ceftazidime or cefotaxime. The availability of cefotaxime as well as ceftazidime strips improves the ability to detect ESBL types which preferentially hydrolyze cefotaxime such as CTX-M-type enzymes [17].

\section{Automated Methods}

Automated methods for bacterial identification and susceptibility testing are used in the detection of ESBL producing organisms. The BD Phoenix System (BectonDickinson Biosciences, Sparks, MD) uses its "expert software" to interpret the growth response to ceftazidime, cefotaxime, ceftriaxone and cefpodoxime, with or without clavulanate. Similarly, the Vitek 2 system (bioMerieux, Marcy L'Etoile, France) uses a card containing ceftazidime and cefotaxime alone and in combination with clavulanate. Ceftazidime or cefotaxime plus betalactamase inhibitors are also used in the MicroScan Walkaway-96 System (Dade Behring, Inc., West Sacramento, CA). The above three semi-automated systems were recently compared to the conventional phenotypic confirmatory tests with regard to their ability to detect ESBL production in well characterized Enterobacteriaceae including Enterobacter spp., Citrobacter freundii and Serratia marcescens. The system with the highest sensitivity was Phoenix (99\%), followed by Vitek 2 (86\%) and MicroScan (84\%) [18].

\section{Implications of Positive Phenotypic Confirmatory Tests}

According to CLSI guidelines, isolates which have a positive phenotypic confirmatory test should be reported as resistant to all cephalosporins (except the cephamycins, cefoxitin, and cefotetan) and aztreonam, regardless of the MIC of that particular cephalosporin. For example, an isolate with a ceftazidime MIC of $>256 \mu \mathrm{g} / \mathrm{ml}$, cefotaxime MIC of $4 \mu \mathrm{g} / \mathrm{ml}$ and cefepime MIC of $2 \mu \mathrm{g} / \mathrm{ml}$, which has a positive phenotypic confirmatory test should be reported as resistant to ceftazidime, cefotaxime and cefepime, when CLSI guidelines are followed. The isolate should also be reported as resistant to penicillins (for example, piperacillin or ticarcillin) regardless of the MIC, but as regard ß-lactam/ß-lactamase inhibitor combinations (for example, ticarcillin/clavulanate or piperacillin-tazobactam) should be reported as susceptible if MICs or zone diameters are within the appropriate range [13].

\section{False Positives and False Negatives Obtained with Phenotypic Confirmatory Tests}

The phenotypic confirmatory tests are highly sensitive and specific compared to genotypic confirmatory tests. However, there are a number of instances whereby the phenotypic confirmatory tests may be falsely positive or negative.

Klebsiella pneumonia or Escherichia coli isolates which lack ESBLs but which hyper produce SHV-1 may give false-positive confirmatory tests. Such isolates can have ceftazidime MICs as high as $32 \mu \mathrm{g} / \mathrm{ml}$ [19]. Characterized one of those organisms where a single base pair change in the promoter sequence resulted in increased production of chromosomally encoded SHV-1. Additionally, outer membrane protein (OMP) analysis revealed a decrease in the quantity of a minor $45-\mathrm{kDa}$ outer membrane protein. There are limited clinical data to determine whether cephalosporin's can be successfully used in patients with such non-ESBL-producing organisms.

Described an outbreak in a paediatric ward in which Klebsiella pneumonia isolates without ESBLs had positive confirmatory tests. The bacteria were subsequently shown to produce TEM-1 and SHV-1 and be deficient in the outer membrane protein OmpK35. Eight bacteraemia patients were successfully treated with third-generation cephalosporins, for which MICs were $1 \mu \mathrm{g} / \mathrm{ml}$ or lower [20].

There are now numerous reports in which Klebsiella pneumonia isolates have been found to harbour plasmidmediated AmpC-type ß-lactamases. Some of these organisms have been found to harbour both AmpC-type ßlactamases and ESBLs. The coexistence of both enzyme types in the same strain results in elevated cephalosporin MICs but may result in false negative tests for the detection of ESBLs. The likely explanation is that AmpCtype ß-lactamases resist inhibition by clavulanate and hence obscure the synergistic effect of clavulanate and cephalosporin's against ESBLs. The relative amount of each ß-lactamase required to obscure the presence of the other is not known [13].

Evaluated the performance of broth micro dilution screening and confirmatory tests at inocula within $0.5 \log$ unit of the standard inoculum [11]. False negative results occurred with both screening and confirmatory tests when lower inocula were used. 


\section{Virology \& Immunology Journal}

\section{Quality Control When Performing Screening and Phenotypic Confirmatory Tests}

Quality control recommendations are that simultaneous testing with a non-ESBL-producing organism as (Escherichia coli ATCC 25922) and an ESBLproducing organism as (Klebsiella pneumonia K6 ATCC 700603) also be performed. Control limits for these organisms are widely available. The ESBL control organism, Klebsiella pneumonia K6 (ATCC 700603), has been recently characterized; it produces SHV-18 and has lost the OmpK35 and OmpK37 porins [4].

\section{Molecular Methods for ESBL Detection}

Early detection of ß-lactamase genes was performed using DNA probes that were specific for TEM and SHV enzymes. The first ESBLs studied were with probes belonging to the TEM family.

Polymerase chain reaction (PCR) with oligonucleotide primers that are specific for a ß-lactamase gene is the easiest and most common molecular method used to detect the presence of a ß-lactamase belonging to a family of enzymes [19]. However, PCR doesn't discriminate between different variants of TEM or SHV [20,21]. Sequencing is essential to discriminate between the nonESBL parent enzymes (eg, TEM1, TEM2, or SHV1) and different variants of TEM or SHV ESBLs (eg, TEM3, SHV2, etc) [22].

Several molecular methods that aid in the detection and differentiation of ESBLs without sequencing have been suggested. The oligotyping method was used to discriminate between TEM-1 and TEM-2. This method used oligonucleotide probes that are designed to detect point mutations under stringent hybridization conditions. Several new TEM variants were identified using this method. These probes are less sensitive for the detection of mutations which are responsible for the extended substrate range [21]. In some cases these mutations lead to the appearance or disappearance of restriction sites. Amplification of the relevant part of the gene by PCR followed by restriction enzyme analysis can thus indicate the presence or absence of specific TEM or SHV derived ESBLs [23].

PCR-single-strand conformation polymorphism (PCRSSCP) has also been applied to the study of ESBLs with satisfactory results [24]. This method has been used to detect a single base mutation at specific location within the beta-lactamase gene. The combination of PCR-SSCP with PCR-restriction fragment length polymorphism (PCR-RFLP) allows the identification of newer SHV variants. The ligase chain reaction (LCR) is used for the identification of SHV genes. Ligase chain reaction (LCR) allows the discrimination of DNA sequences that differ by a single base pair [25].

A sequence-specific peptide nucleic acid (PNA)-based multiplex PCR detection method provides an accurate means of identification of bla (GES-2) compared to the standard PCR and the gene sequencing techniques [26].

In Italy, the distribution of PER-1 ESBL was investigated by southern blot analysis with a PER-1 genespecific probe [27]. However, nucleotide sequencing remains the standard for determination of the specific $ß$ lactamase gene present in a strain [28].

\section{References}

1. Moland E, Kim S, Hong S, Kenneth S (2008) Newer $\beta$ Lactamases: Clinical and laboratory implications, Part II. Clin Microbiol Newsletter 30(10): 79-85.

2. Ramphal R, Ambrose PG (2006) Extended-spectrum $\beta$-lactamases and clinical outcomes: Current data. Clin Infect Dis 42: 164-172.

3. Ben Ami RMJ, Schwaber S, Navon Venezia S, Schwartz D, Giladi M, et al. (2006) Influx of extended-spectrum $\beta$-lactamase-producing Enterobacteriaceae into the hospital. Clin Infect Dis 42(7): 925-934.

4. Clinical and Laboratory Standard Institute (2009) Performance standards for antimicrobial susceptibility testing: Nineteenth informational supplement M100-S19. Wayne, PA, USA.

5. Paterson DL, Bonomo RA (2005) Extended spectrum $\beta$-lactamases: A clinical update. Clin Microbiol Rev 18(4): 657-686.

6. Ho PL, Chow KH, Yuen KY, Ng WS, Chau PY (1998) Comparison of a novel, inhibitor-potentiated discdiffusion test with other methods for the detection of extended-spectrum beta-lactamases in Escherichia coli and Klebsiella pneumoniae. J Antimicrob Chemother 42(1): 49-54.

7. Thomson KS, Sanders CC (1997) A simple and reliable method to screen isolates of Escherichia coli and Klebsiella pneumonia for the production of TEM and SHV derived extended-spectrum beta-lactamases. Clin Microbiol Infect 3(5): 549-554. 
8. $\quad$ Oliver A, Weige LM, Rasheed JK, McGowan JE, Raney $P$, et al. (2002) Mechanisms of decreased susceptibility to cefpodoxime in Escherichia coli. Antimicrob Agents Chemother 46(12): 3829-3836.

9. Casals J, Pringler N (1990) Detection in the routine laboratory of new plasmid-mediated broad-spectrum $\beta$-lactamases in Enterobacteriaceae. $7^{\text {th }}$ Mediterranean Congress of Chemotherapy, Barcelona, Spain.

10. Schooneveldt JM, Nimmo GR, Giffard $P$ (1998) Detection and characterisation of extended spectrum beta-lactamases in Klebsiella pneumoniae causing nosocomial infection. Pathology 30(2): 164168.

11. Queenan AM, Foleno B, Gownley C, Wira E, Bush K (2004) Effects of inoculum and beta-lactamase activity in AmpC- and extended-spectrum betalactamase (ESBL)-producing Escherichia coli and Klebsiella pneumoniae clinical isolates tested by using National Committee for Clinical Laboratory Standards ESBL methodology. J Clin Microbiol 42(1): 269-275.

12. Jarlier V, Nicolas MH, Fournier G, Philippon A (1988) Extended broad-spectrum beta-lactamases conferring transferable resistance to newer beta-lactam agents in Enterobacteriaceae: Hospital prevalence and susceptibility patterns. Rev Infect Dis 10(4): 867-878.

13. Perez F, Endimiani A, Hujer KM, Bonomo RA (2007) The Continuing Challenge of ESBLs. Curr Opin Pharmacol 7(5): 459-469.

14. Ho PL, Chan WM, Tsang KW, Wong SS, Young K (2002) Bacteremia caused by Escherichia coli producing extended-spectrum beta-lactamase: A case-control study of risk factors and outcomes. Scand J Infect Dis 34(8): 567-573.

15. Bedenic B, Randegger C, Boras A, Haechler H (2001) Comparison of five different methods for detection of SHV extended-spectrum beta-lactamases. J Chemother 13(1): 24-33.

16. Shahid M, Malik A, Agrawal M, Singhal S (2004) Phenotypic detection of extended-spectrum and AmpC ß-lactamases by a new spot-inoculation method and modified three-dimensional extract test: Comparison with the conventional three-dimensional extract test. J Antimicrob Chemother 54(3): 684-687.

\section{Virology \& Immunology Journal}

17. Stürenburg E, Sobottka I, Noor D, Laufs R, Mack D (2004) Evaluation of a new cefepime-clavulanate ESBL E-test to detect extended-spectrum betalactamases in an Enterobacteriaceae strain collection. J Antimicrob Chemother 54(1): 134-138.

18. Rice LB, Carias LL, Hujer AM, Bonafede M, Hutton R, et al. (2000) High-level expression of chromosomally encoded SHV-1 beta-lactamase and an outer membrane protein change confer resistance to ceftazidime and piperacillin-tazobactam in a clinical isolate of Klebsiella pneumoniae. Antimicrob. Agents Chemother 44(2): 362-367.

19. Wu TL, Siu LK, Su LH, Lauderdale TL, Lin FM, et al. (2001) Outer membrane protein change combined with co-existing TEM-1 and SHV-1 beta-lactamases lead to false identification of ESBL-producing Klebsiella pneumoniae. J Antimicrob Chemother 47(6): 755-761.

20. Fluit AC, Visser MR, Schmitz FJ (2001) Molecular Detection of Antimicrobial Resistance. Clin Microbiol Rev 14(4): 836-871.

21. Bradford PA (2001) Extended spectrum betalactamases in the 21st century: Characterization, epidemiology and the detection of this important resistance threat. Clin Microbiol Rev 14(4): 933-951.

22. Garza Ramos U, Martínez Romero E, Silva Sánchez J (2007) SHV-type extended spectrum $\beta$-lactamase (ESBL) are encoded in related plasmids from enterobacteria clinical isolates from Mexico. Salud Publica Mex 49(6): 415-421.

23. Subramaniam G, Palasubramaniam $S$, Navaratnam $P$ (2006) SHV-5 extended-spectrum $\beta$-lactamases in clinical isolates of E.coli in Malaysia. Indian J Med Microbiol 24(3): 205-207.

24. Kim J, Lee HJ (2000) Rapid discriminatory detection of genes coding for SHV beta-lactamases by ligase chain reaction. Antimicrob Agents Chemother 44(7): 1860-1864.

25. Wiegand I, Geiss HK, Mack D, Sturenburg E, Seifert H, et al. (2007) Detection of extended-spectrum betalactamases among Enterobacteriacea by use of semiautomated microbiology systems and manual detection procedures. J Clin Microbiol 45(4): 11671174. 


\section{Virology \& Immunology Journal}

26. Pagani L, Mantengoli E, Migliavacca R, Nucleo E, Pollini S, et al. (2004) Multifocal detection of multidrug-resistant Pseudomonas aeruginosa producing the PER-1 extended-spectrum $\beta$-lactamase in northen Italy. J Clin Microbiol 42(6): 2523-2529.
27. Al Naiemi N, Schipper K, Duim B, Bart A (2006) Application of minimal sequence quality values prevents misidentification of the blaSHV type in single bacterial isolates carrying different SHV extended-spectrum- $\beta$-lactamase genes. J Clin Microbiol 44(5): 1896-1898. 